\title{
Influence of ion irradiation on temperature dependent electrical transport behavior of thin graphite flakes
}

\author{
JABRIL A. KHAMAJ*
}

Faculty of Mechanical Engineering, College of Engineering, Jazan University, Jazan 45142, Kingdom of Saudi Arabia

\begin{abstract}
In this article, the influence of ion irradiation on temperature dependent electrical transport characteristics of thin graphite flakes was investigated. Thin graphite flakes were exfoliated by mechanical exfoliation method. Scanning electron microscopy was used to study surface morphology of the graphite flakes. The resistance versus temperature studies revealed that the graphite flake not subjected to $\mathrm{Ga}^{+}$ion-irradiation showed a perfect metallic behavior, while the graphite flake after ion-irradiation showed a semiconducting behavior. The current-voltage (I-V) characteristics of bare and ion-irradiated graphite flakes were investigated. The bare graphite flake showed an ohmic-type I-V characteristics representing metallic behavior, while the ionirradiated graphite flake showed a non-linear type diode-like characteristics. The temperature-dependent conductance measurements of ion-irradiated graphite flake were also performed and discussed in detail. The effect of $\mathrm{Ga}^{+}$ions on the electronic transport behavior of thin graphite flakes has been discussed based on the investigation results.
\end{abstract}

Keywords: ion-irradiation; electronic transport; thin graphite flake; temperature dependency

(C) Wroclaw University of Technology.

\section{Introduction}

In recent decades, the research on carbon family materials has received great attention of the world's scientific community [1, 2]. Especially graphite, graphene, and carbon nanotubes have been used successfully for various applications in sensors, transistors, light emitting diodes, and high-temperature applications. Graphite is a three-dimensional material which has a sheet-like layered-structure. Carbon atoms are arranged in a plane and the interlayers are weakly bonded vertically with van der Waals forces [3]. Hence, thin layers of graphite (graphene, in case of monolayer graphite) can easily be exfoliated from the bulk graphite using mechanical exfoliation method. These days, graphene is considered as a suitable alternative material for silicon electronics [4-6]. Each graphite sheet has a hexagonal carbon arrangement with strong $\sigma$ bonding $\left(\mathrm{sp}^{2}\right)$ in the ab-plane while perpendicular $\pi$-orbital electrons along the c-axis are responsible for ab-plane conductivity [7].

\footnotetext{
*E-mail: jabrilkhamaj2015@gmail.com
}

Recently, the scientific interest on graphite has been revived by the electronic transport and magnetization results of Highly Oriented Pyrolytic Graphite (HOPG) as the carrier transport in graphite is known as two-dimensional [8,9]. The electronic transport behavior shows remarkable technological and scientific potential [10]. The in-plane (ab) transport of graphite reveals a metallic behavior as expected, however the outof-plane transport (across the vertical planes) is still a controversial issue [11-13]. At room temperature, graphite exhibits electrical anisotropy in which the in-plane conductance was reported as $\sim 10^{4} \Omega^{-1} \cdot \mathrm{cm}^{-1}$ and very low out-of-plane conductance as $\sim 10 \quad \Omega^{-1} \cdot \mathrm{cm}^{-1}$ [14]. However, according to the report by Natori et al. [15], the very large anisotropy ratio $\left(\rho_{\mathrm{c}} / \rho_{\mathrm{a}} \sim 10^{5}\right)$ has been found in bulk HOPG at room temperature.

It has also been observed that when the temperature is increased, the anisotropy of resistivity decreases resulting in complicated electronic transport characteristics of graphite [16]. Despite many reports on bulk graphite conducted for several years, the transport properties are still not well recognized and not well understood. Also 
the fabrication of graphite based mesoscopic devices, such as tunnel junctions, nanopatterns, nanostacks using ion-beam, e-beam lithography has become popular in past few years $[13,17,18]$. These graphite based mesoscopic devices with novel properties induced a special focus on the research of few-layer or multilayer graphite samples as they can be used as promising candidates for spintronics, micro nano-devices and resonators $[8,19,20]$. Ion beam irradiation over the graphite samples may affect the function and properties of these devices. Hence, it is very crucial to understand the effect of ion-beam irradiation on the properties of graphite. There are many reports on electronic properties of bulk graphite. However, only few studies have been reported so far on ionirradiated graphite, particularly temperature dependent electronic transport studies have not been performed yet because the size and geometry of the devices should be small in this type of experiments.

In this report, we investigate the temperature dependent transport properties of a thin graphite flake before and after ion-radiation. In order to irradiate the graphite flake, graphite samples were put inside a chamber and subjected to irradiation by a focused ion beam. The effect of ion beam irradiation on the electronic properties of thin graphite flake has been discussed.

\section{Materials and methods}

In this experiment, HOPG material for preparing thin graphite flakes on $\mathrm{Si} / \mathrm{SiO}_{2}$ substrates was used. Prior to depositing the graphite flakes, the substrates were cleaned with acetone followed by ultrasonic bath cleaning. The thickness of $\mathrm{SiO}_{2}$ layer on $\mathrm{Si}$ substrate was $\sim 300 \mathrm{~nm}$. Mechanical peeling-off technique was used to prepare thin graphite flakes. After several times of repeated peeling-off, thin graphite flakes were identified by using an optical microscope and were transferred on the substrate. Silver paste was used to deposit electrical contacts which were then annealed at $200{ }^{\circ} \mathrm{C}$ to lower the contact resistance. Gallium $\left(\mathrm{Ga}^{+}\right)$ions were irradiated on the thin graphite flake for $30 \mathrm{~s}$.
This was performed inside the chamber with focused ion beam (FIB). Low- and roomtemperature electrical transport measurements were performed using cryo-cooler (helium compressor unit) in which the temperature could be lowered to $50 \mathrm{~K}$.

\section{Results and discussion}

Fig. 1 presents a scanning electron microscopy image (SEM) of thin graphite flake exfoliated from the bulk HOPG. The thin graphite flake with smooth surface morphology has been identified and chosen for electrode contact making. The silver contacts were made what is clearly seen in the SEM picture.

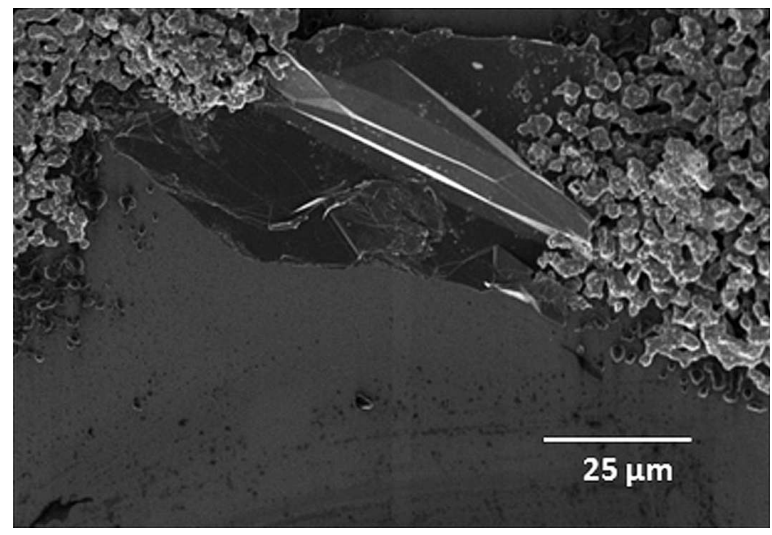

Fig. 1. SEM image of thin graphite flake with silver electrodes before irradiation (image scale bar is $25 \mu \mathrm{m})$.

Fig. 2 shows the temperature dependent electrical characteristics of a thin graphite flake before (a) and after ion-beam irradiation (b). The resistance (R) versus temperature (T) plot of the thin graphite flake before ion irradiation is shown in Fig. 2a. A perfect metallic behavior is observed. When the temperature is decreased to $50 \mathrm{~K}$, the resistance of the graphite flake is gradually decreased. It is believed that the impurity-assisted interlayer hopping conduction mechanism combined with carrier scattering is the main factor responsible for this metallic characteristics of graphite [21]. However, the ion-irradiated graphite flake shows an anomalous transport behavior. 

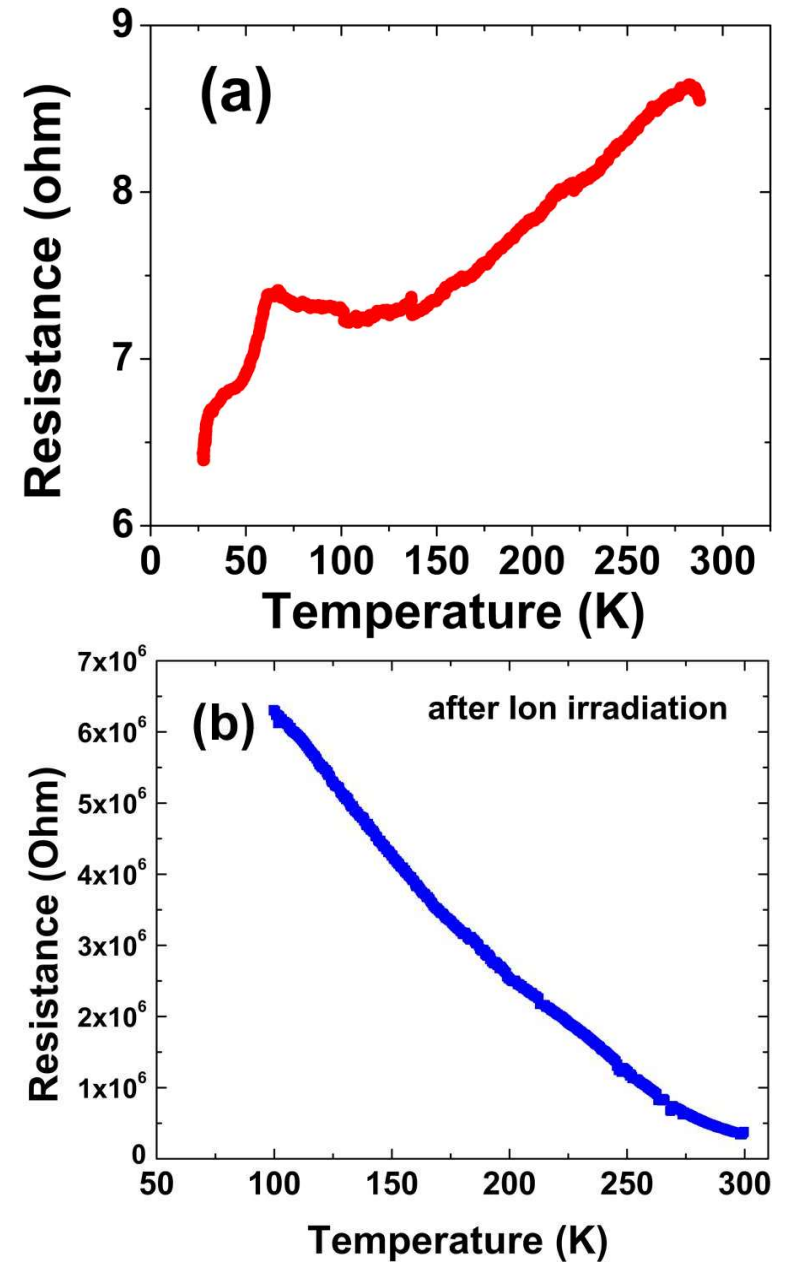

Fig. 2. R-T behavior of thin graphite flake (a) before ion irradiation (b) after ion irradiation.

Semiconducting behavior was observed when the temperature was gradually decreased to $50 \mathrm{~K}$. This has been shown in Fig. 2b. Due to ion irradiation, the semiconducting nature was induced in the graphite flake. When the temperature was decreased to $50 \mathrm{~K}$, the in-plane resistance of the ion-irradiated thin graphite flake showed a surprisingly large increase in resistance value (in the range of $\mathrm{M} \Omega$ ). The semiconducting behavior might have arisen due to the thermally excited carriers which are responsible for this behavior observed above $50 \mathrm{~K}$ [22], however, in our case, it is strongly believed that the irradiated $\mathrm{Ga}^{+}$ions on the graphite flake might be the main source of resistance increase in the sample which resulted in the semiconducting transport behavior.
The current versus voltage (I-V) characteristics of a thin graphite flake is presented in Fig. 3. The $\mathrm{I}-\mathrm{V}$ characteristics were measured at room temperature. A perfect ohmic behavior is observed (Fig. 3a) for the thin graphite flake which exhibits the metallic nature. However, after ion-irradiation, the I-V behavior is changed into semiconducting characteristics. The non-linear type I-V behavior is observed even at $300 \mathrm{~K}$ for various current inputs (from $25 \mu \mathrm{A}$ to $150 \mu \mathrm{A}$ ) which is represented in Fig. 3b.

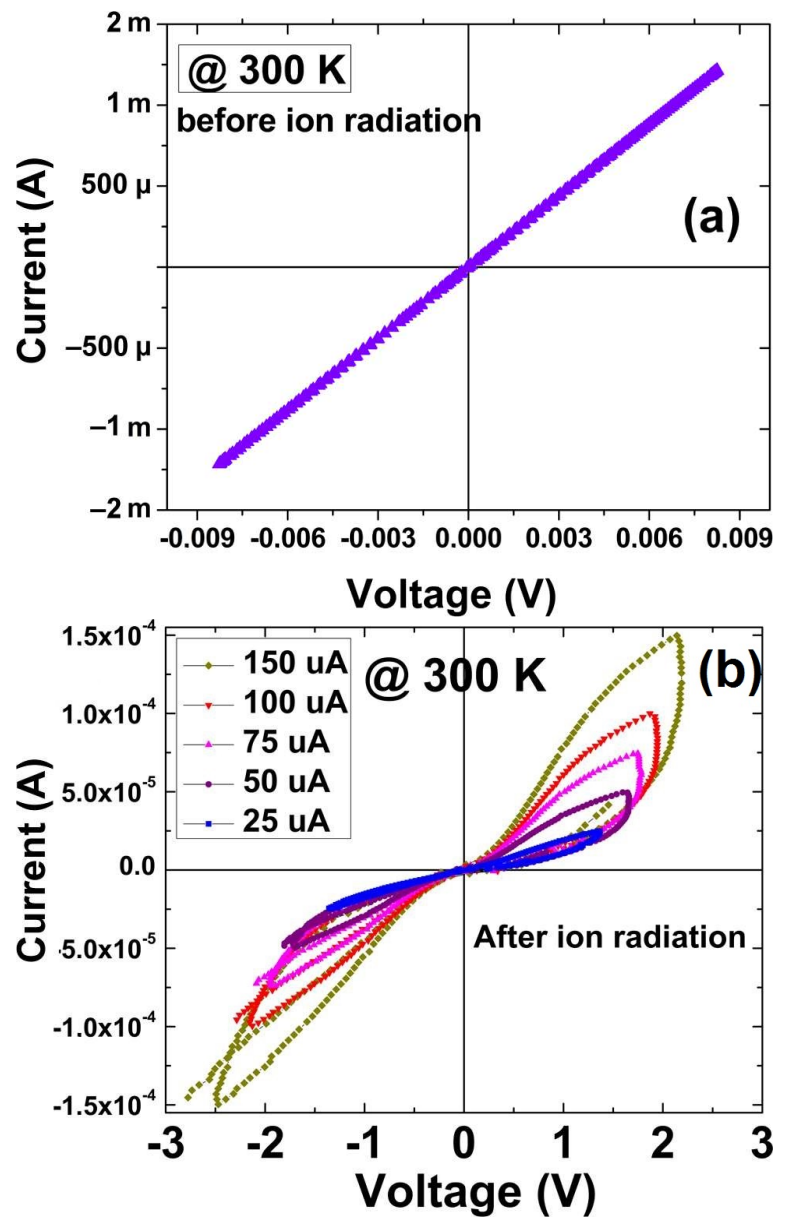

Fig. 3. Room temperature current-voltage (I-V) characteristics of a thin graphite flake (a) before ion irradiation (b) after ion irradiation.

When the input current is increased, the width of the non-linear loop also gets increased. The nonlinear I-V characteristics is also a typical evidence for the semiconducting nature of the ion-irradiated thin graphite flake. 
In Fig. 4, the temperature-dependent I-V characteristics are shown for ion-irradiated thin graphite flake. The I-V characteristics reveal a nonlinear and diode-like behavior at $300 \mathrm{~K}$ and at $50 \mathrm{~K}$, respectively. When the temperature goes down, the diode-like behavior has appeared. The appearance of diode-like current-voltage characteristics at $50 \mathrm{~K}$ might be attributed to the restricted charge carrier tunneling between the interlayers of graphite which results in high resistance at low temperature [22].

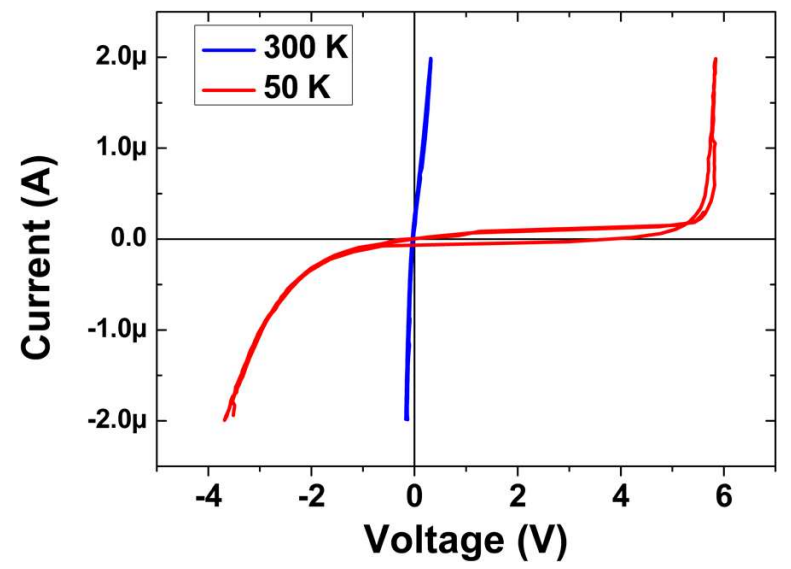

Fig. 4. Temperature dependent current-voltage characteristics of a thin graphite flake after ion irradiation.

In order to observe the temperature dependent electronic transport phenomenon in the ionirradiated graphite flake, temperature dependent conductance measurement was performed, which is presented in Fig. 5. At $300 \mathrm{~K}$, the sample exhibits a significant conductance even at positive and negative voltage bias. We observed the conductance of $\sim 22 \mu \mathrm{S}$ at negative bias, however, it was decreased to $5 \mu \mathrm{S}$ when the sample was positively biased. At $50 \mathrm{~K}$, a larger conductance (approximately $5 \mathrm{mS}$ ) at both biases was measured.

This abrupt change in conductance might mainly be attributed to the suppression of resistance caused by $\mathrm{Ga}^{+}$ions (irradiated on the sample) at low temperature. At low temperature, the semiconducting nature of $\mathrm{Ga}^{+}$ions reduced which might lower the sample resistance.

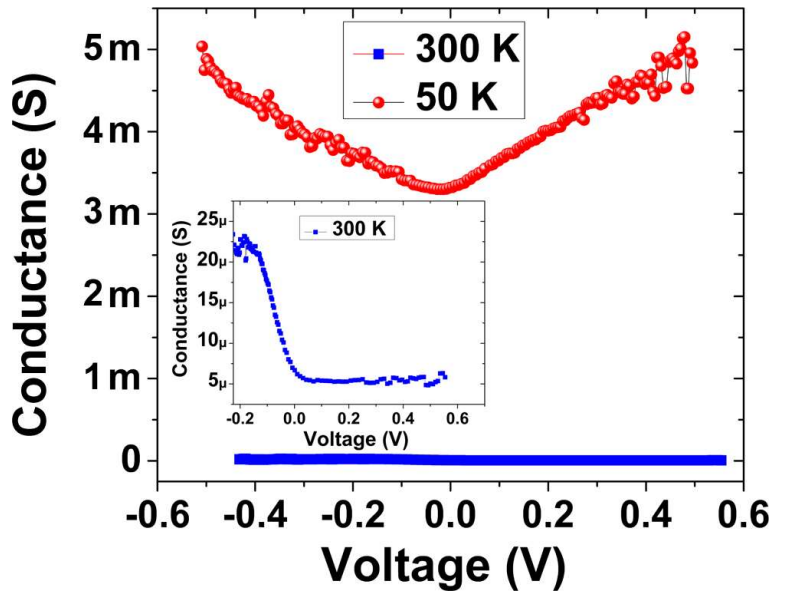

Fig. 5. Temperature dependent conductance measurement of a thin graphite flake after ion irradiation.

\section{Conclusions}

In this work, the influence of ion-irradiation on temperature dependent electrical transport behavior of thin graphite flakes was investigated. The graphite flake not subjected to ion-irradiation revealed a perfect metallic behavior while the ionirradiated graphite flake showed the semiconducting characteristics. This behavior was further confirmed through the current-voltage characteristics which were measured at $300 \mathrm{~K}$ and $50 \mathrm{~K}$. The nonlinear type diode-like characteristics was observed for an ion-irradiated thin graphite flake. In addition, our conductance measurements results further confirmed that the ion-irradiated graphite flake showed a higher conductance at $50 \mathrm{~K}$ when compared to the conductance measured at $300 \mathrm{~K}$. This has been mainly due to the suppression of resistance caused by $\mathrm{Ga}^{+}$ions at low-temperature. We believe that our investigation results give a good insight into the electronic transport behavior of a thin graphite flake when it is subjected to ion-irradiation.

\section{Acknowledgements}

The author expresses his sincere gratitude to the Jazan University and the President of the Jazan University, His Excellency Professor Mohammed Bin Ali Rubai'ya, for his constant encouragement towards research and development in the Jazan University. 


\section{References}

[1] Kopelevich Y., Torres J.H.S., DA Silva R.R., Mrowka F., Kempa H., Esquinazi P., Phys. Rev. Lett., 90 (2003), 156402.

[2] LuK'Yanchuk I.A., Kopelevich Y., Phys. Rev. Lett., 93 (2004), 166402.

[3] Kelly B.T., Physics of graphite, Applied Science, London, Englewood, 1981, pp 267 - 361.

[4] Javey A., Jing G., Qian W., Lundstrom M., DAI H., Nature, 424 (2003), 654.

[5] Wind S.J., APPEnZeller J., Martel R., Derycke V., Avouris P., Appl. Phys. Lett., 80 (2002), 3817.

[6] Tans S.J., Verschueren R.M., Dekker C., $\mathrm{Na}$ ture, 393 (1998), 49.

[7] BanerjeE S., SARdar M., Gayathri N., Tyagi A.K., Baldev RAJ., Appl. Phys. Lett., 88 (2006), 062111.

[8] Kopelevich Y., Esquinazi P., Torres J., Moenlecke S., J. Low Temp. Phys., 119 (2000), 691.

[9] Kempa H., Solid State Commun., 115 (2000), 539.

[10] Berger C., Song Z.M., Li T.B., Li X.B., OGBAZghi A.Y., FENG R., DAI Z.T., MARChENKOV A.N., Conrad E.H., First P.N., DE HeEr W.A., J. Phys. Chem. B, 108 (2004), 19912.

[11] Morelli D.T., Uher C., Phys. Rev. B, 30 (1984), 1080.
[12] Uher C., Hockey R.L., Jacob E.B., Phys. Rev. B, 35 (1987), 9.

[13] Novoselov K.S., Geim A.K., Morozov S.V., JiAng D., Dubonos S.V., GrigorieVA I.V., Firsov A.A., Science, 306 (2004), 666.

[14] Dresselhaus M.S., Dresselhaus G., Adv. Phys., 30 (1981), 139.

[15] NAtori A., J. Phys. Soc. Jpn., 55 (1986), 4370.

[16] LI X., Doctor Thesis, Submitted to Georgia Institute of Technology, 2008.

[17] Leduc H.G., Bumble B., Cypher S.R., Judas A.J., Stern J.A., Proceedings of the Third International Symposium on Space Terahertz Technology, University of Michigan, Ann Arbor, 1992., p. 408.

[18] LU K., J. Nanosci. Nanotechnol. 9 (2009), 2598.

[19] BUNCH J.S., VAN DER ZANDE A.M., VERBRIDGE S.S., Frank I.W., TANenbaum D.M., PARPia J.M., Craighead H.G., Mceuen P.L., Science, 315 (2007), 490.

[20] Novoselov K.S., Jiang Z., Zhang Y., MoroZOV S.V., Stormer H.L., Zeitler U., MaAN J.C., Boebinger G.S., Kim P., Geim A.K., Science, 315 (2007), 1379.

[21] Kopelevich Y., Squinazi P., Adv. Mater., 19 (2007), 4559.

[22] Sugihara K., Phys. Rev. B, 37 (1988), 4752.

Received 2015-11-08

Accepted 2016-03-08 